m09.p05

\section{Merging crystallography, site-directed mutagenesis and molecular modelling to unravel the regulatory mechanism of photosynthetic GAPDH}

$\underline{\text { S. Fermani }}{ }^{\mathrm{a}}$, F. Sparla ${ }^{\mathrm{b}}$, G. Falini ${ }^{\mathrm{a}}$, L. Martellic, M. Zaffagnini ${ }^{\mathrm{b}}$, A. Ripamonti ${ }^{\mathrm{a}}$, R. Casadio ${ }^{\mathrm{c}}$, P. Pupillo $^{\mathrm{b}}$, P. Trost $\mathrm{t}^{\mathrm{b}}$.

a Dipartimento di Chimica "G. Ciamician", Università di Bologna Alma Mater Studiorum, via Selmi 2, 40126 Bologna, Italy ${ }^{b}$ Laboratorio di Fisiologia molecolare delle piante, Dipartimento di Biologia Evoluzionistica Sperimentale, Università di Bologna Alma Mater Studiorum, via Irnerio 42,40126 Bologna, Italy. ${ }^{c}$ Laboratorio di Biocomputing, Università di Bologna Alma Mater Studiorum, via San Giacomo 9/2, 40126 Bologna, Italy.

\section{Keywords: protein crystallography, molecular modelling, enzyme structure function}

Photosynthetic glyceraldehyde-3-phosphate dehydrogenase (GAPDH) is involved in the carbon reduction cycle common to every organism displaying oxygenic photosynthesis. This metabolic cycle is finely regulated by environmental conditions and thioredoxins play a major role in this respect. The full understanding of the regulatory mechanism does require the knowledge of GAPDH tridimensional structure, both in reduced and oxidized form. Two types of almost identical GAPDH subunits (GapA and GapB) do exist in plants. Subunit GapB exceeds GapA by a C-terminal extension (CTE) of 30 amino acids including the cysteine pair responsible for redox regulation. The subunits are arranged into isoforms with either $\mathrm{A}_{4}$ or $\mathrm{A}_{\mathrm{n}} \mathrm{B}_{\mathrm{n}}$ stochiometry. Photosynthetic GAPDH displays dual coenzyme specificity with preference for NADPH over NADH. The NADH-dependent activity is constitutive while any regulatory property of the enzyme exclusively regard the NADPH-activity. Tetrameric GapA has been crystallized in complex with either NADP or NAD [1,2] and the molecular basis of coenzyme recognition have been satisfactorily explained. Residues Thr33 and Ser188 are involved in NADP over NAD selectivity by interacting with 2'-phosphate of NADP. Mutants of recombinant spinach GapA $\left(\mathrm{A}_{4}-\mathrm{GAPDH}\right)$ with $\mathrm{Thr} 33$ or Ser188 replaced by Ala were produced [3].

Native $A_{2} B_{2}$-GAPDH could only be crystallized in complex with NADP under oxidizing conditions. However, while the structure of the tetramer has been solved, the actual position of the CTE could not be determined due to the lack of electron density. Minor differences between the structures of oxidized $\mathrm{A}_{2} \mathrm{~B}_{2}-\mathrm{GAPDH}$ (inhibited) and $\mathrm{A}_{4}-\mathrm{GAPDH}$ (activated) provide hints to understand the structural basis of regulation and have been tested by site-directed mutagenesis. The C-terminal domain of GAPDH B has been modelled.

[1] S. Fermani, A. Ripamonti, P. Sabatino, G. Zanotti, S. Scagliarini, F. Sparla, P. Trost, and P. Pupillo J. Mol. Biol. 2001, 314(3), 537-552.

[2] G. Falini, S. Fermani, A. Ripamonti, P. Sabatino, F. Sparla, P. Pupillo, and P. Trost Biochemistry 2003, 42(16), 4631-4639.

[3] F. Sparla, S. Fermani, G. Falini, A. Ripamonti, P. Sabatino, P. Pupillo and P. Trost J. Mol. Biol. 2004, 340(5), 1025-1037.

\section{m09.p06}

\section{Structure of Pteridine Reductase (PTR1) from Trypanosoma brucei}

P.K. Fyfe, A. Dawson, F. Gibellini, K. McLuskey, N. Sienkiewicz, A.H. Fairlamb, W.N. Hunter

School of Life Sciences, University of Dundee, Dow Street, Dundee, DD1 5EH, U.K.E-mail: p.k.fyfe@dundee.ac.uk

\section{Keywords: enzyme inhibitor design, biological macromolecules, folate dependent enzymes}

Anti-folate resistance in the trypanosomatid parasites is due in part to pterin reductase (PTR1) which is capable of reducing folate. This allows uptake of folate even when the primary enzyme, dihydrofolate reductase, is inhibited, and makes PTR1 an important drug target. The crystal structure of PTR1 from Trypanosoma brucei complexed with the cofactor NADPH and the inhibitor methotrexate has been determined to $2.2 \AA$. The protein structure is closely related to the previously determined L. major structure [1], with the cofactor and inhibitor bound in a similar fashion. The methotrexate molecule is significantly better defined in the T. brucei structure but there is no indication of increased MTX-protein interaction. A nonconservative Leu-Cys substition close to the active side is observed.

[1] Gourley D.G., et al., Nature Str. Biol., 2000, 8, 521-525. 